\title{
Germanica
}

\section{Siegfried Kracauer : « Le caractère de la propagande totale »}

Introduction au texte

\section{Guillaume Plas}

\section{OpenEdition \\ Journals}

Édition électronique

URL : https://journals.openedition.org/germanica/8508

DOI : 10.4000/germanica.8508

ISSN : 2107-0784

\section{Éditeur}

Université de Lille

\section{Édition imprimée}

Date de publication : 1 juin 2020

Pagination : 31-36

ISBN : 978-2-913857-45-2

ISSN : 0984-2632

Référence électronique

Guillaume Plas, "Siegfried Kracauer : «Le caractère de la propagande totale » », Germanica [En ligne], 66 | 2ème trimestre 2020, mis en ligne le 02 janvier 2022, consulté le 08 janvier 2022. URL : http:// journals.openedition.org/germanica/8508; DOI : https://doi.org/10.4000/germanica.8508 


\title{
Siegfried Kracauer : « Le caractère de la propagande totale »
}

\author{
Introduction au texte
}

Guillaume Plas

Universität Freiburg

Totalitäre Propaganda, l'étude de Siegfried Kracauer dont nous présentons ici la traduction de l'une des parties, en guise de préambule à sa traduction exhaustive, nous parvient à double titre sous la forme d'une œuvre inachevée. Sa version définitive, typoscrite, est non seulement demeurée inédite du vivant de Kracauer, mais elle a, de plus, été par la suite perdue. Son manuscrit « brut », originel, en est donc l'unique version conservée. Elle l'est, sous une forme extrêmement ardue à déchiffrer (du fait d'un mélange d'écritures différentes, dont des abbréviations non univoques, d'un système complexe de renvois internes, et de la très grande quantité de modifications apportées au texte), dans trois cahiers de format A5 déposés au Literaturarchiv de Marbach.

Ces aspérités du document sont à l'image de son histoire, particulièrement sinueuse. Et ce dès avant sa genèse à proprement parler ; car pour différentes raisons, son auteur n'était au départ pas le moins du monde disposé à l'écrire pour ce qui allait être son destinataire - la Zeitschrift für Sozialforschung, dirigée alors par Horkheimer - ; quant à ce destinataire, il n'était guère enclin à accepter de publier quelque texte que ce soit de cet auteur ${ }^{1}$. Pourtant, à la fin de l'année 1936, et grâce au travail

1. - Nous suivons dans notre reconstruction succincte de la genèse de ce texte le dossier préparé par l'éditeur du manuscrit (Bernd Stiegler, « Nachwort : Eine 
d'intermédiaire d'Adorno, Kracauer, qui tentait alors par tous les moyens de trouver un lieu hors d'Allemagne vers lequel sa femme et lui puissent émigrer, se résolut à travailler pour l'Institut für Sozialforschung alors déjà domicilié à New York, et l'Institut, à accepter de lui offrir de le publier. Entre la fin de l'année 1936 et mars 1937, les deux parties s'entendent ainsi sur la rédaction d'une étude consacrée à « la genèse, la fonction et la structure de la propagande national-socialiste $»^{2}$.

Kracauer, espérant obtenir via ce travail la possibilité d'être intégré de façon pérenne à l'Institut et donc d'enfin émigrer à son tour, s'y jette à corps perdu et envoie dès la fin de l'année 1937 une première partie du manuscrit, de 105 pages, suivie, au début de l'année 1938, d'une deuxième partie d'encore 50 pages. Or, tandis qu'Horkheimer semble avoir été satisfait de l'étude, abstraction faite de sa taille (qui dépassait très largement la taille maximale d'une publication au sein de sa revue), Adorno fait rapidement savoir autour de lui qu'il est effaré par la piètre qualité analytique et les errements idéologiques qu'il déclare y percevoir. Se sentant à cet égard pleinement conforté dans le sentiment qu'il avait eu l'occasion de partager à peine quelques mois plus tôt avec Walter Benjamin au sujet de la monographie consacrée par Kracauer à Jacques Offenbach, dont la lecture l'avait plongé dans « le plus pénible des embarras $»^{3}$, il énumère dans le Gutachten au vitriol rédigé à l'attention d'Horkheimer les défauts de ce nouvel écrit : une méthodologie faite d'« analogies plus ou moins vagues », des développements constellés d'« improvisations et de naïvetés théoriques », et, surtout, un auteur qui tout simplement « ne dispose pas d'une formation de marxiste $»^{4}$. Dès lors, la sentence qu'il avait formulée un an et demi plus tôt en

Gesprächsnotiz », in : Siegfried Kracauer, Totalitäre Propaganda, Berlin, Suhrkamp, 2013, p. 309-329) et, surtout, le chapitre consacré par Jörg Später à cet épisode dans sa récente biographie de Kracauer (« Das "Institut für Sozialfälschung” », in : Jörg Später, Siegfried Kracauer : eine Biographie, Berlin, Suhrkamp, 2016, p. 373-383), auquel nous renvoyons le lecteur pour davantage de renseignements. On se référera également, en français, aux pages consacrées à ce texte dans l'étude d'Olivier Agard, Kracauer. Le chiffonier mélancolique, Paris, CNRS Editions, 2010, p. 220-235, ainsi que, du même auteur, le tout récent ouvrage Les écrits de Kracauer sur la propagande / Kracauers Schriften zur Propaganda, Conférences Franz Hessel, édité par / herausgegeben von Stephan Braese \& Céline Trautmann-Waller, Paris, Editions de l'éclat, 2019.

2. - Lettre de Kracauer à Horkheimer du 28 janvier 1937, citée d'après B. Stiegler, " Nachwort : Eine Gesprächsnotiz », op. cit., p. 320 (" die Genesis, Funktion und Struktur der nationalsozialistischen Propaganda $»)$.

3. - Lettre d'Adorno à Benjamin du 4 mai 1937, citée d'après J. Später, Siegfried Kracauer, op. cit., p. 331.

4. - Theodor W. Adorno, « Gutachten über die Arbeit "Die totalitäre Propaganda Deutschlands und Italiens", S. 1 bis S. 106, von Siegfried Kracauer » [5 mars 1938], in : S. Kracauer, Totalitäre Propaganda, op. cit., p. 262-265, citations resp. p. 262, 264 et 262 (« [...] mehr oder minder vagen Analogien », « [...] theoretischer Improvisation und Naivetäten », « [...] daß Kracauer kein geschulter Marxist ist »). 
reprenant une proposition de Benjamin - « il faudrait le [Kracauer, G.P.] "placer sous tutelle", c'est-à-dire que si nous voulons le sauver, au sens littéral et au sens intellectuel du terme, alors n'allons-nous pouvoir le faire que contre son gré $»^{5}$ - devait désormais être mise à exécution. Chargé par Horkheimer d'apporter au texte de Kracauer les modifications nécessaires à sa publication, Adorno en reprit bien plutôt le peu qui lui convenait - et fit, avec ces quelques bribes éparses, du Adorno : surlignant la tonalité résignée des constats politiques et sociologiques, radicalisant et généralisant les éléments dialectiques des phénomènes observés, modifiant enfin les accents herméneutiques et, par suite, les conclusions interprétatives ${ }^{6}$. Kracauer ne pouvait bien évidemment accepter un tel procédé, par lequel « de [s] on langage, aucune phrase n'[était] restée intacte $\gg^{7}$, et refusa l'imprimatur ; l'étude ne parut donc jamais dans la revue de l'Institut, ni, d'ailleurs, dans aucun autre organe de publication de son vivant, malgré les efforts qu'il fit ultérieurement en ce sens. D'une particulière violence symbolique, cet épisode peut à

5. - Lettre d'Adorno à Horkheimer du 12 octobre 1936, citée d'après J. Später, Siegfried Kracauer, p. 377 (« man müsste ihn "entmündigen”, d. h. wenn man ihn retten will im buchstäblichen und intellektuellen Sinn, so wird man es nur gegen ihn selber können »).

6. - Donnons ici un seul exemple de cette modification de ton argumentatif comme (et de manière intimement liée) de constat analytique. Tandis que Kracauer s'en tient dans l'ensemble de son texte à la dénonciation des satisfactions apparentes que fournit la propagande totalitaire, dont il souligne de façon répétée qu'elle est qualitativement distincte de la réclame au sein d'un système capitaliste notamment par son nihilisme à l'égard des citoyens qu'elle berne et du concept de vérité qu'elle foule des pieds, Adorno modifie ce constat de la manière suivante (et nous laissons exceptionnellement cette citation dans l'original allemand pour mieux faire entendre sa 'voix' particulière) : " Manche der Propagandaaktionen sind als Fassadenphänomene leicht noch durchschaubar. Wenn von beiden Eingängen der Villa die Schilder "Herrschaften" und "Bediente" entfernt werden, die Türen jedoch unverändert an ihrer Stelle bleiben, weiss jeder sehr wohl, wo er hingehört. Aber von solchen Massnahmen, die mehr der sadistischen Wut gegens gute Leben als dem Hass gegen die Ungerechtigkeit entspringen, führt eine stetige Linie zu den grossen propagandistischen Institutionen, deren Scheinbefriedigungen von den wirklichen kaum mehr zu unterscheiden sind, weil die wirklichen nicht besser sind als sie selber. » (Siegfried Kracauer, « Zur Theorie der autoritären Propaganda [von Theodor W. Adorno erstellte Kurzfassung] », in : S. Kracauer, Totalitäre Propaganda, p. 266-296, ici p. 276 ; nous soulignons, G.P.) De la même façon que l'on peut raisonnablement penser que si Kracauer a finalement attribué à la propagande qu'il étudiait le qualificatif de « totalitaire » et non celui de « totale », c'est afin de mieux la distinguer de celle d'autres régimes (cf. infra notre remarque à ce sujet), il était totalement étranger à son intention de démonstration de niveler ainsi, dans un geste au contraire typiquement adornien, les « satisfactions apparentes » offertes au sein d'un régime totalitaire et celles « réelles » offertes au sein d'un régime non-totalitaire, que ne séparait plus dans l'abject une différence qualitative mais, tout au plus, une différence quantitative, de simple degré.

7. - Lettre à Horkheimer du 19 avril 1940 (en français dans l'original), citée d'après B. Stiegler, « Nachwort : Eine Gesprächsnotiz », op. cit., p. 326. 
n'en pas douter être considéré comme « le plus douloureux de l'histoire des relations de Kracauer avec le cercle de Horkheimer $»^{8}$.

Les conséquences de cette histoire mouvementée du texte sur la forme sous laquelle celui-ci nous parvient devaient être préservées dans la traduction de la partie présentée ici. Nous y avons donc volontairement reproduit les quelques imperfections (des répétitions de mots et autres inélégances stylistiques, ainsi que des redites dans l'argumentation, notamment), que Kracauer a peut-être éradiquées avant de rendre son typoscrit, ou qu'il aurait sans nul doute éradiquées au plus tard lors de la mise au point de la version finale. Elles n'entravent de toute façon nullement la clarté de son propos.

Cette partie $\mathrm{B}$ dont nous proposons la traduction est la deuxième de sept parties de taille similaire d'un texte qui, dans la publication allemande de 2013, compte 145 pages. Dans le corps du manuscrit conservé, les sept parties ne portent aucun titre ; mais si l'on en croit une « disposition » notée par Kracauer dans l'un de ses carnets bien avant l'achèvement de sa rédaction (entre le 10 et le 13 juillet 1937), le plan de l'étude, qui s'appelait alors encore Die totale Propaganda, a, au moins pendant un temps, été le suivant :
A. Die Genesis der totalen Propaganda
B. Der Charakter der totalen Propaganda
C. Die Methode der totalen Propaganda
D. Die Propaganda auf dem Weg zur Macht
E. Die totale Propaganda als Instrument der Macht ${ }^{9}$.

Les titres respectifs des deux dernières parties, $\mathrm{F}$ et $\mathrm{G}$, qui correspondent vraisemblablement au deuxième ensemble ajouté ultérieurement et envoyé distinctement, au début de l'année 1938, ne sont en revanche indiqués à aucun endroit des trois cahiers conservés, et demeurent donc vierges dans la version publiée.

L'élément de titulation le plus important à relever, néanmoins, est la modification de poids apportée au titre général de l'étude, puisque Kracauer explique dans une lettre à Horkheimer du 24 janvier 1938 s'être décidé à changer son adjectif et avoir finalement opté pour Die totalitäre Propaganda. Ein politischer Traktat ${ }^{10}$. Nous ne nous étendrons

8. - O. Agard, Siegfried Kracauer. Le chiffonnier mélancolique, op. cit., p. 220.

9. - [A. La genèse de la propagande totale; B. Le caractère de la propagande totale ; C. La méthode de la propagande totale; D. La propagande totale sur le chemin vers le pouvoir ; E. La propagande totale comme instrument du pouvoir.] « Disposition », in : S. Kracauer, Totalitäre Propaganda, p. 244-258, ici p. 244.

10. - Cf. B. Stiegler, « Nachwort : Eine Gesprächsnotiz », op. cit., p. 323. Notons au passage que le choix effectué par Stiegler d'un titre légèrement différent pour la publication du manuscrit chez Suhrkamp - Totalitäre Propaganda, sans sous-titre - 
pas ici sur les raisons qui ont pu amener Kracauer à opter pour l'adjectif « totalitaire » à la place de « total ». Qu'il soit néanmoins relevé que ce choix apparaît, d'une part, parfaitement cohérent si l'on se réfère au texte manuscrit, puisqu'il y est fait nettement plus souvent usage du premier adjectif que du second. Il semble, d'autre part, et comme nous l'avons déjà brièvement indiqué, également pertinent du point de vue de l'intention de démonstration de l'auteur, puisqu'il lui permettait de mieux marquer la ligne de partage entre les systèmes totalitaires et les systèmes non-totalitaires qui, néanmoins, peuvent parfaitement employer eux aussi une propagande totale : distinguo essentiel, et qui allait constituer, nous l'avons dit, l'un des points centraux de la discorde entre Adorno et lui au sujet de la validité de ses analyses.

Comme la disposition de mai 1937 le laisse deviner, Kracauer a choisi une approche aussi exhaustive que possible pour étudier la propagande totalitaire. Son attention se porte tout autant sur les nationaux-socialistes et les fascistes qui en font usage que sur ses effets sur les groupes sociaux qui en sont la cible. Et il mobilise et associe, à cette fin, des éléments interprétatifs d'ordre aussi bien historique (l'importance de la défaite de 1918) que sociologique (l'impossibilité de cantonner le nationalsocialisme et le fascisme à une classe particulière ou à un groupe social particulier, ces mouvements regroupant autant des bourgeois que des prolétaires, des propriétaires terriens que des ouvriers) et politique (le maintien d'une distinction nette entre ces idéologies et mouvements et le communisme d'un côté via le critère du nihilisme, et le capitalisme de l'autre côté, qui prospère quant à lui au mieux au sein d'un régime libéral). Enfin, l'apport de la psychologie et de la psychanalyse (pour expliquer le comportement des individus soumis à une autorité de ce genre) est peut-être le témoin le plus univoque de l'inscription, parfois intrinsèquement motivée, parfois davantage stratégique, de l'étude dans le milieu idéel de la Théorie critique. Il importe néanmoins de noter à ce sujet que la catégorie sociologique de référence y est, autant que la classe, la «masse » (un concept qui, significativement, faisait d'ailleurs partie du titre de la toute première mouture du texte, un « exposé » encore intitulé Masse und Propaganda ${ }^{11}$ ). Car si l'usage du concept de classe permet de mettre en évidence l'impossibilité de concilier des intérêts antagonistes et, par suite, si l'on aspire à créer une " communauté du peuple », la nécessité d'une manipulation, qui incombe au travail de propagande, seul celui de masse, dans l'indistinction qu'il exprime, permet quant à lui de rendre compte de façon tout à fait exacte de cette propagande, dont l'un des effets repose sur le nombre, ainsi que de faire ressortir au mieux

n'est nulle part légitimé (ni même, du reste, explicité) dans l'appareil critique qu'il adjoint au texte lui-même.

11. - Cf. cet exposé in S. Kracauer, Totalitäre Propaganda, op. cit., p. 231-237. 
l'idéologie totalitaire dans la radicalité de son anti-individualisme. Dès lors, force est de donner d'une certaine manière raison à Adorno dans ses doutes émis quant à la rigueur du marxisme de Kracauer; et une simple lecture superficielle du texte permet en effet de constater que celui-ci, tout en étant en réalité parfaitement identifiable comme d'obédience marxiste, n'en demeure pas moins dans quelques passages bien sentis d'une iconoclastie prononcée : ainsi dans l'hypothèse prudemment avancée dans la partie ici traduite (mais, lue à huit décennies de distance, parfaitement crédible, voire tout bonnement confirmée) selon laquelle le système capitaliste pourrait fort bien, après tout, " s'imaginer peut-être seulement être en danger, à force de se l'entendre dire ».

Une part importante de l'étude consiste toutefois moins en une analyse à proprement parler qu'en une stricte " phénoménologie » (le terme était revendiqué par Kracauer, tandis qu'il fut repris, mais à des fins critiques, par Adorno dans son Gutachten) des outils et modes d'action de cette propagande. Tactique de «l'image en miroir » consistant à accuser l'adversaire des fautes que l'on commet afin de les commettre d'autant plus impunément, mise à nu publique assumée de ses propres stratégies propagandistiques pour mieux s'assurer du degré d'hypnose atteint par les masses à sa merci, impertinence totale de l'idée au profit d'un activisme de la propagande dont l'idée n'est plus que l'outil presque aléatoirement choisi... : à cet égard, c'est à la description d'une véritable post-factualité avant la lettre - et de ses conséquences sur le « mode d'existence civilisé »-que Kracauer procède ainsi. Et c'est peut-être en celle-ci - qu'il nous soit permis ici d'émettre pour finir et à titre d'hypothèse ce jugement, à rebours de celui d'Adorno - que nous paraît résider le plus grand, non moins que le plus surprenant enrichissement qu'offre ce texte de 1937 à ses lecteurs d'aujourd'hui. 